\title{
The Rapidly Changing Treatment Landscape of Systemic Therapy for HER-2 Positive Metastatic Breast Cancer
}

\author{
Larissa Russo-Vorms ${ }^{1}$, Christoforos Astaras ${ }^{1 *}$, José Sandoval ${ }^{1}$, Maxime Borgeaud ${ }^{1}$, Alexandre Bodmer ${ }^{1}$
}

\section{ABSTRACT}

Human epidermal growth factor receptor 2 (HER-2) overexpressed breast cancer has traditionally been considered as an aggressive disease with high risk of systemic and brain metastases and poor prognosis. Nowadays, we have many HER-2 targeting treatments that have radically changed patients' outcomes. Among the oldest ones, trastuzumab, pertuzumab, lapatinib and trastuzumab-emtansine have been for some years the backbone of established therapies. New drugs, such as tucatinib, trastuzumab-deruxtecan, pyrotinib, neratinib and margetuximab, have recently joined the therapeutic arsenal, revolutionizing the therapeutic field of this disease. Many other drugs are currently under development and give hope, with encouraging results to heavily pretreated patients or those with central nervous system metastasis.

Keywords: breast cancer, epidermal growth factor receptor (EGFR), human epidermal growth factor receptor 2 (HER-2), systemic therapy, tyrosine kinase inhibitors (TKIS)

\section{INTRODUCTION}

Breast cancer is a heterogeneous disease with multiple molecular characteristics and clinical behavior depending on the histological background. Human epidermal growth factor receptor 2 (HER-2) gene amplification resulting in overexpression of the HER-2 protein can be detected in 15\%-20\% of invasive breast tumors and is associated with aggressive tumor behavior and poor survival rate. ${ }^{1,2}$ In the last thirty years, the introduction of therapies inhibiting HER-2 pathway have altered the treatment paradigm of this disease, leading to unprecedented survival outcomes. ${ }^{3}$ Tyrosine kinase inhibitors (TKIs), monoclonal antibodies and antibody-drug conjugates compose the backbone of the treatment of HER-2 metastatic breast cancer disease. Defining the best sequencing among all those treatment options as well as understanding the complicated resistant mechanisms, remains a challenge. This comprehensive review summarizes the therapeutic approaches based on the relevant pivotal trials (Figure 1, Table 1).
Department of Oncology University Hospital of Geneva (HUG) Geneva, Switzerland

* Corresponding author: Dr Christoforos Astaras Department of Oncology University Hospital of Geneva (HUG) Geneva, Switzerland Email: Christoforos.Astaras@hcuge.ch DOI: 10.36000/hbT.OH.2021.09.044 ISSN: 2673-2092 (Print) and 2673-2106 (Online)

This article was received on August 10, 2021.

This article was accepted on September 17, 2021.

This article was published on October 15, 2021.

Russo-Vorms L, Astaras C, Sandoval J, Borgeaud M, Bodmer A. The Rapidly Changing Treatment Landscape of Systemic Therapy for HER-2 positive Metastatic Breast Cancer. healthbook TIMES Onco Hema. 2021;(9):16-23.

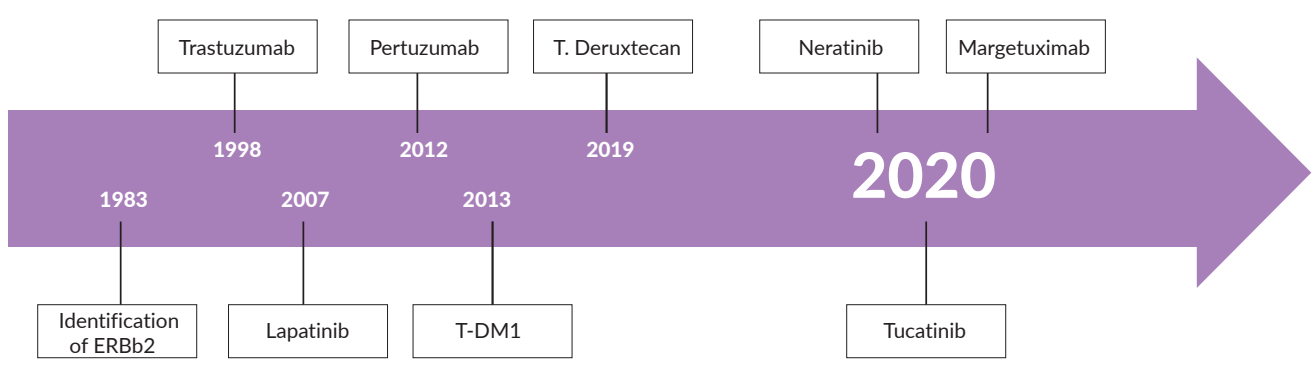

Figure 1. Milestones of principal anti-HER-2 breast cancer therapies. HER-2, human epidermal growth factor receptor 2; T-DM1, trastuzumab-emtansine; ERBb2, Erb-B2 Receptor Tyrosine Kinase 2 (=HER2). 


\section{CLASSIC STANDARD OF CARE}

HER-2 amplification, found in approximately $15 \%-20 \%$ of breast cancers ${ }^{4}$, is a tyrosine kinase receptor of the erythroblastic leukemia viral oncogene homologue (ERBB) receptors family. Like other members of the ERBB family, HER-2 is a transmembrane protein composed of an extracellular domain, a transmembrane segment and an intracellular domain with a tyrosine kinase function. ${ }^{5}$ HER-2 can dimerize either with another HER-2 receptor (autodimerization), or with another member of the ERBB family (principally HER-3) (heterodimerization). Of note, HER-2 dimerization occurs independently of any ligand binding and HER-2 has no known ligand. Dimerization leads to the activation of the intracellular kinase domain and subsequently intracellular signaling cascades, such as the RAS/RAF/MAP kinase and the PIK3CA/ $\mathrm{AKT} / \mathrm{mTOR}$ pathways, ultimately leading to cell proliferation.

Trastuzumab is a humanized monoclonal antibody targeting the extracellular domain of HER-2, which acts by several mechanisms. Trastuzumab induces the internalization of the HER-2 receptor and inhibits the activation of intracellular signaling pathways. Moreover, it promotes the immune response towards tumor cells by attracting natural killer cells and allowing their recognition of tumor cells via its Fragment crystallizable $(\mathrm{Fc})$-segment, enhancing their destruction ${ }^{6}$. In the early 2000s, the combination of trastuzumab with chemotherapy showed superiority to chemotherapy alone in metastatic HER-2 positive breast cancer, with an absolute overall survival (OS) benefit of approximately 5 months. ${ }^{7}$ The addition of an HER-2 blockade to various treatment regimens has consistently been shown to improve outcome, with an absolute OS of 5-8 months in a meta-analysis (HR: 0.82 [95\% CI: 0.71-0.94]; $\mathrm{p}=0.004$ ). ${ }^{8}$ Interestingly, in the study of Slamon et al. (2001), the association of trastuzumab and anthracycline led to $16 \%$ of cardiac toxicity and was therefore abandoned, in favor of the association of trastuzumab with a mono-chemotherapy, frequently a taxane.

It has been suggested that vinorelbine could be an alternative therapeutic option for taxanes in this setting, demonstrating comparatively similar progression-free survival (PFS) (12.4 months vs 15.3 months, HR: 0.94 [95\% CI: 0.71-1.25]; $\mathrm{p}=0.67)$ and $\mathrm{OS}$ rates (35.7 months vs 38.8 months, HR: 1.01 [95\% CI: $0.71-1.42$ ]; $\mathrm{p}=0.98)$ in a phase III trial. ${ }^{9}$

\begin{tabular}{|c|c|c|c|}
\hline Study & Phase & $\begin{array}{l}\text { Line of } \\
\text { treatment }\end{array}$ & $\begin{array}{l}\text { Results } \\
\text { (only statistically significant mentioned) }\end{array}$ \\
\hline $\begin{array}{c}\text { CLEOPATRA: } \\
\text { chemotherapy }+ \\
\text { trastuzumab }+ \text { pertuzumab } \\
\text { vs chemotherapy }+ \\
\text { trastuzumab }\end{array}$ & III & 1 & $\begin{array}{l}\text { PFS: } 18.7 \text { months vs } 12.4 \text { months } \\
\text { OS: } 56.5 \text { months vs } 40.8 \text { months }\end{array}$ \\
\hline $\begin{array}{c}\text { EGF104900: } \\
\text { trastuzumab + lapatinib vs } \\
\text { lapatinib alone }\end{array}$ & III & 2 & $\begin{array}{l}\text { PFS: } 11.1 \text { weeks vs } 8.1 \text { weeks } \\
\text { OS: } 14 \text { months vs } 8.3 \text { months }\end{array}$ \\
\hline $\begin{array}{c}\text { EMILIA: } \\
\text { T-DM1 vs chemotherapy }+ \\
\text { lapatinib }\end{array}$ & II & 2 & $\begin{array}{l}\text { PFS: } 9.6 \text { months vs } 6.4 \text { months } \\
\text { OS: } 30.9 \text { months vs } 25.1 \text { months }\end{array}$ \\
\hline $\begin{array}{c}\text { NALA: } \\
\text { chemotherapy + neratinib } \\
\text { vs chemotherapy }+ \\
\text { lapatinib }\end{array}$ & III & 3 & $\begin{array}{l}\text { PFS: } 8.8 \text { months vs } 6.6 \text { months } \\
\text { OS: statistically non-significant }\end{array}$ \\
\hline $\begin{array}{c}\text { HER2CLIMB: } \\
\text { chemotherapy }+ \\
\text { trastuzumab + tucatinib } \\
\text { vs chemotherapy }+ \\
\text { trastuzumab }\end{array}$ & III & 3 & $\begin{array}{l}\text { PFS: } 7.8 \text { months vs } 5.6 \text { months } \\
\text { OS: } 21.9 \text { months vs } 17.4 \text { months }\end{array}$ \\
\hline $\begin{array}{c}\text { DESTINY-Breast03 } \\
\text { trastuzumab } \\
\text { deruxtecan }\end{array}$ & III & 2 & $\begin{array}{l}\text { PFS: not reached vs } 6.8 \text { months } \\
\text { OS: immature }\end{array}$ \\
\hline $\begin{array}{l}\text { SOPHIA: } \\
\text { margetuximab + } \\
\text { chemotherapy } \\
\text { vs trastuzumab + } \\
\text { chemotherapy }\end{array}$ & III & $>2$ & $\begin{array}{l}\text { PFS: } 5.8 \text { months vs } 4.9 \text { months } \\
\text { OS: ongoing (2021) }\end{array}$ \\
\hline $\begin{array}{c}\text { Pyrotinib + chemotherapy } \\
\text { vs lapatinib + } \\
\text { chemotherapy }\end{array}$ & II & $>2$ & $\begin{array}{c}\text { PFS: } 18 \text { months vs } 7 \text { months } \\
\text { OS: premature data/statistically non-significant }\end{array}$ \\
\hline
\end{tabular}

Table 1. Table showing the trials having established the main anti-HER-2 treatments. OS, overall survival; PFS, progression-free survival; T-DM1, trastuzumab-emtansine; vs, versus 
The SAKK $22 / 99^{10}$, a phase III study, randomized patients to receive either trastuzumab alone followed by trastuzumab plus chemotherapy at disease progression or upfront trastuzumab in combination with chemotherapy. Time to progression (TTP) and OS did not differ significantly between the two groups (12.2 months vs 10.3 months, HR: 0.7 [95\% CI: $0.5-1.1] ; \mathrm{p}=0.1$ and 35.6 months vs 36.3 months, HR: 0.9 [95\% CI: $0.6-1.5] ; \mathrm{p}=0.55$, respectively). In the subgroup analysis of the trastuzumab alone arm, patients without the visceral disease had a significantly longer TTP compared with the patients with visceral metastases (21.8 months vs 10.1 months; HR: 2.1 [95\% CI: 1.1-4.2]; $\mathrm{p}=0.03$ ). Based on these findings, the authors suggested that single-agent trastuzumab could be a sufficient treatment option for individual patients without visceral involvement.

Pertuzumab, another anti-HER-2 antibody, targets a different epitope of the extracellular domain of the protein and specifically inhibits the HER-2/HER-3 heterodimerization, which normally leads to strong activation of intracellular signaling pathways. Currently, based on the results of the CLEOPATRA trial, the association of trastuzumab and pertuzumab with a taxane represents the standard first-line treatment for metastatic HER-2 positive breast cancer. In this phase III, randomized controlled trial, patients underwent randomization to receive either trastuzumab-pertuzumab and docetaxel or trastuzumab-docetaxel alone. ${ }^{11}$ Patients in the intervention group had an absolute OS benefit of 16 months ( 57 months vs 41 months; HR: 0.68 [95\% CI: 0.56-0.84]; $\mathrm{p}<0.001$ ), with more than $1 / 3$ of the patients still alive at 8 years. Toxicities were higher in the trastuzumab-pertuzumab group, with diarrhea, rash and hematological toxicities.

Although the CLEOPATRA trial used docetaxel, other taxanes constitute a valid alternative, based on the results of the PERUSE study. This trial compared trastuzumabpertuzumab in association with docetaxel, paclitaxel or nab-paclitaxel, at the investigator's choice. ${ }^{12}$ Among 1,436 patients, different taxanes showed similar median PFS when associated with trastuzumab-pertuzumab: 19.6 months (95\% CI: 16.9-21.8) for docetaxel, 23 months (95\% CI: 19.8-25.8) for paclitaxel, and 18.1 months (95\% CI: 12.2-32.3) for nab-paclitaxel. Limitations of this study are that patients were not randomized between the different taxanes and that only 65 patients received nab-paclitaxel. Different toxicity profiles may guide the choice between these agents, as paclitaxel is associated with a higher risk of neuropathy compared with docetaxel (31\% vs $16 \%$ ) but a lower risk of febrile neutropenia ( $1 \%$ vs $11 \%$ ) or mucositis ( $14 \%$ vs $25 \%$ ).

In patients with hormone receptor-positive tumors, regimens avoiding chemotherapy by combining trastuzumab with or without pertuzumab and aromatase inhibitors (AI) have been studied. Of note, none of these have been head-to-head compared with the association of chemotherapy and anti-HER2. The association of trastuzumab and an AI increased PFS when compared with an AI, but with rather disappointing results (5.6 months vs 3.8 months, HR: 0.63 [95\% CI: 0.47-0.84]). ${ }^{13}$ The association of trastuzumab-pertuzumab and an AI has been studied in the PERTAIN study ${ }^{14}$, which demonstrated superiority in PFS for the triple association over trastuzumab plus an AI (18.9 months vs 15.8 months, HR: 0.65 [95\% CI: $0.48-0.89$ ]; $\mathrm{p}=0.007)$. Interestingly, $>50 \%$ of the patients in the PERTAIN study had received induction chemotherapy. This strategy could be considered in situations in which chemotherapy is not an option or as maintenance therapy.

Trastuzumab-emtansine or T-DM1, an antibody drugconjugate, consists of the antibody trastuzumab, bound to the cytotoxic molecule emtansine, an inhibitor of microtubules. When binding to HER-2, T-DM1 is internalized, and emtansine is released within the tumor cell, leading to delivery of the cytotoxic drug directly on its target. ${ }^{15} \mathrm{~T}$-DM1 was tested in the first-line metastatic setting, combined with or without pertuzumab, in comparison to trastuzumab associated to a taxane in the MARIANNE study. ${ }^{16}$ T-DM1, with or without pertuzumab, failed to demonstrate a significant benefit in PFS compared to trastuzumab with taxane. Its use is therefore not recommended for the first-line treatment of metastatic disease, except for patients with progressive disease occurring during or soon after (approximately 6 months -12 months) the end of adjuvant treatment with pertuzumab and trastuzumab. ${ }^{17}$

T-DM1 demonstrated benefits in the second or third-line setting in the EMILIA ${ }^{18}$ and TH3RESA ${ }^{19}$ studies. In the pivotal EMILIA trial, 978 patients with metastatic HER-2 positive breast cancer who had progressed on prior treatment with trastuzumab and taxane were randomized between T-DM1 or capecitabine and lapatinib. T-DM1 improved both PFS (10 months vs 6 months, HR: 0.65 [95\% CI: 0.55-0.77]; $\mathrm{p}<0.0001)$ and OS (31 months vs 25 months; HR: 0.75 [95\% CI: $0.64-0.88]$ ) and resulted in less grade 3-4 adverse events ( $41 \%$ vs $57 \%)$.

The TH3RESA trial compared T-DM1 to the investigator's choice chemotherapy in the third-line setting, for patients previously treated with trastuzumab and lapatinib-based regimens. Of note, $68 \%$ of the controlled group also received trastuzumab along with chemotherapy. Among the 602 patients treated, T-DM1 resulted in a better PFS (6.2 months vs 3.3 months, HR: 0.53 [95\% CI: 0.42-0.66]) and OS (22.7 months vs 15.8 months; HR: 0.68 [95\% CI: 0.54-0.85]; $\mathrm{p}=0.0007$ ), and comparable safety profiles T-DM1 represents the treatment of choice in the second or third-line setting, for patients who have progressed on one or two trastuzumab-based regimens.

Lapatinib, a reversible inhibitor of HER-2 and epidermal growth factor receptor -1 (EGFR-1), represents the first TKI approved for the treatment of HER-2 positive metastatic breast cancer. Lapatinib is a small molecule targeting and inhibiting the intracellular kinase domain of the HER-2 
receptor. ${ }^{20}$ In association with capecitabine, lapatinib resulted in an amelioration of PFS (6.2 months vs 4.3 months; HR: 0.57 [95\% CI: 0.43-0.77]; $\mathrm{p}<0.001)$ when compared with capecitabine alone, in the EGF 100151 trial, among patients previously treated with trastuzumab, a taxane and an anthracycline. ${ }^{21}$ However, the trial was stopped early after the preliminary interim analysis, as no survival benefit could be demonstrated.

Lapatinib has also been tested alone or in association with trastuzumab in the EGF104900 trial ${ }^{22}$, with 291 patients previously treated with trastuzumab. The trastuzumablapatinib association improved OS compared to lapatinib alone (14 months vs 8.3 months, HR: 0.74 [95\% CI: 0.57-0.97]; $\mathrm{p}=0.026$ ), mainly demonstrating the benefit of continuing trastuzumab beyond progression. Based on these results and the results of the EMILIA trial, lapatinib represents one of the multiple options responsible for the third-line settings after progression on trastuzumab-pertuzumab-taxane and T-DM1.

\section{NEW THERAPEUTIC AGENTS}

Trastuzumab-deruxtecan represents another antibody-drug conjugate, consisting of a monoclonal antibody targeting HER-2 coupled with deruxtecan, a topoisomerase I inhibitor. While stable in the plasma, trastuzumab-deruxtecan is easily cleaved by enzymes upregulated in cancer cells, such as lysosomal cathepsins, releasing the cytotoxic compound deruxtecan. Of note, trastuzumab-deruxtecan has a high cytotoxic drug to antibody ratio $(8: 1)$, compared to $\mathrm{T}_{-} \mathrm{DM} 1^{23}$. Moreover, deruxtecan shows high permeability to the cell membrane and can diffuse to bystander cells, regardless of their expression of HER-2. ${ }^{24}$ Trastuzumab-deruxtecan has also demonstrated activity against HER-2 low-tumor cells. ${ }^{25}$

Efficacy of trastuzumab-deruxtecan in metastatic breast cancer was studied in the phase II Destiny-Breast01 trial ${ }^{26}$, a single-arm trial. Participants were heavily pretreated patients that had received a median of 6 prior lines of treatment for metastatic disease. All patients had received prior treatment with trastuzumab and T-DM1, and the majority (65\%) had received pertuzumab. An impressive overall response rate (ORR) of 60.9\% (95\% CI: 53.4-68.0) was reported in this population, with a median duration of response of 14.8 months (95\% CI: 13.8-16.9) and a median PFS of 16.4 months (95\% CI: 12.7 to not reached). Of note, some significant toxicities were observed, including hematological toxicities nausea, vomiting, and interstitial lung disease (13.6\%). Adverse events led to treatment discontinuation in $15 \%$ of patients. Noteworthy, most interstitial lung diseases were of low grade, but 2 deaths were attributed to interstitial lung disease by independent adjudication. Based on these results, the Food and Drug Administration (FDA) granted accelerated approval to trastuzumab deruxtecan for metastatic HER-2 positive breast cancer for the patients with at least 2 prior anti-HER-2 regimens for metastatic disease.
The results of the phase III Destiny-Breast03 trial, evaluating trastuzumab-deruxtecan in the second-line setting have been presented recently at the ESMO Congress 2021. ${ }^{27}$ Overall, 524 patients previously treated with trastuzumab and a taxane were randomized to either trastuzumab-deruxtecan or T-DM1. Trastuzumab-deruxtecan significantly improved PFS (the primary endpoint) compared with T-DM1 (not reached vs 6.8 months; HR: 0.28 [95\% CI: 0.21-0.37]; $\left.\mathrm{p}=7.8 \times 10^{-22}\right)$. The estimated 12 -month OS rates seemed to favor trastuzumab-deruxtecan, but the pre-specified boundary for statistical significance was not reached ( $94.1 \%$ vs $85.9 \% 12$ months OS), likely due to immature follow-up. Regarding safety, treatment-emergent adverse event rates were similar between both arms, with interstitial lung disease occurring in $10.5 \%$ of patients treated with trastuzumab-deruxtecan (grade 1-2: $9.7 \%$, grade $3: 0.7 \%$, grade $4-5: 0 \%)$. Thus, the pulmonary toxicity profile seemed significantly lower than what was observed in previous trials in more heavily pretreated patients. These results will likely be practice-changing, establishing trastuzumab-deruxtecan as the preferred therapy in the second-line setting.

Trastuzumab-deruxtecan is currently being compared to treatment of investigator's choice (trastuzumab-capecitabine or capecitabine-lapatinib) in phase III randomized controlled trial Destiny-Breast $02^{28}$, in patients having received T-DM1. Trastuzumab-deruxtecan is also being tested in the second-line setting against T-DM1 in the Destiny-Breast03 trial ${ }^{29}$ and more recently in the first-line for metastatic disease in the Destiny-Breast $09^{30}$, in which trastuzumab deruxtecan, in association, or not, with pertuzumab, is compared to trastuzumab, pertuzumab and a taxane. Based on preliminary results with promising signs of activity in HER-2 low metastatic breast cancer, trastuzumab deruxtecan is also currently evaluated in this setting ${ }^{31,32}$.

Tucatinib. Brain metastases remain a major issue for patients with metastatic HER-2 positive breast cancer since up to half of them will develop intracranial lesions. ${ }^{33}$ Until recently, treatment possibilities were radiation on metastasis or surgery, with existing antitumoral drugs (trastuzumab emtansine or the association of capecitabine with lapatinib) ${ }^{34}$, showing only a modest efficacy with substantial toxicities. Tucatinib is an oral, reversible, powerful and selective HER-2 TKI. In cell signaling tests, tucatinib was found to be 1,000 times more selective for HER-2 than for the EGFR, with the great advantage of brain penetrance.

It has been studied as a third-line therapy of metastatic HER-2 positive breast cancer in the HER2CLIMB trial ${ }^{35}$, as a component of dual blockade in association with trastuzumab and capecitabine and compared to the latter two drugs alone. Patients with and without active brain disease were included. The tucatinib arm offered an increase of the PFS at 1 year (33.1\% vs $12.3 \%$, HR: 0.54 [95\% CI: $0.42-0.71$ ] ; $p<0.001$ ), as well as an OS benefit at 2 years ( $44.9 \%$ vs $26.6 \%$, HR: 0.66 


\section{healthbook TIMES Oncology Hematology}

[95\% CI: 0.50-0.88]; $\mathrm{p}=0.005)$. The median PFS benefit was of 2.2 months and the median OS benefit was of 4.5 months. The superiority of tucatinib was shown independently of the presence of visceral disease or the hormonal receptor status. Particularly with patients with brain metastases, tucatinib showed efficacy with a confirmed objective response of $40.6 \%$ (95\% CI: 35.3-46.0). The estimated PFS at 1 year was $24.9 \%$ in the tucatinib-combination group and $0 \%$ in the placebocombination group (HR: 0.48 [95\% CI: 0.34-0.69]; $\mathrm{p}<0.001$ ), with the risk of disease progression or death being $52 \%$ lower in the tucatinib-combination group compared to the standard arm.

Concerning safety profile, the most common adverse event was diarrhea, more frequent in the intervention group but mostly of grade 1 or 2 . The major grade 3 adverse event was palmar-plantar erythrodysesthesia which was more frequent in the tucatinib group (13\% vs $9 \%$ ). However, there was no major difference in discontinuation rate in both arms of the trial.

These results confirm that tucatinib is an interesting and innovative option for heavily pretreated patients, especially those with brain metastases. The FDA has already approved the drug, although the main limitation in clinical practice is still its high cost. ${ }^{36}$ Currently, in Switzerland, tucatinib is not one of the drugs approved by the Federal Office of Public Health (FOPH) and first, a negotiation with the patient's health insurance is necessary.

A trial studying the combination of tucatinib with trastuzumab deruxtecan in patients having received at least 2 prior lines of anti-HER-2 treatment is currently ongoing. ${ }^{37}$

Neratinib is an irreversible HER-1, 2, and 4 TKI. It was first studied as adjuvant treatment in the EXTENET trial, including high-risk patients with HER-2 overexpressed disease in a localized setting after 1 year of adjuvant trastuzumab ${ }^{38}$, in which it reduced significantly the proportion of clinically relevant distant and locoregional breast cancer relapses.

The NALA trial evaluated this pan-HER-2 inhibitor as a third-line treatment for patients with metastatic HER-2 breast cancer, including those with asymptomatic intracranial disease. Neratinib was associated with capecitabine and compared to lapatinib and capecitabine (and not the actual standard of care, trastuzumab). ${ }^{39}$ After a median follow-up of 30 months, the PFS was increased by 2 months in the neratinib combination group (8.8 months vs 6.6 months HR: 0.76 [95\% CI: $0.63-0.93$ ]; stratified log-rank $\mathrm{p}=0.0059$ ), with no statistical difference in OS. The overall response was similar in both arms, with a median duration of response prolonged by 3 months in the intervention group (HR: 0.50 [95\% CI: 0.33-0.74]; $\mathrm{p}=0.0004)$. Concerning safety, the most common adverse events were diarrhea and nausea in the neratinib group, as seen in the adjuvant setting with no impact on the discontinuation rate. A subgroup analysis showed better results in patients with non-visceral or hormone-negative disease. Of note, treatment with neratinib with patients presenting brain metastases showed no difference in PFS compared to the control arm.

Neratinib is approved by FDA in this setting, but it is actually difficult to implement it in daily practice because of the negative cost-efficacy ratio. Neratinib has also been studied in the first-line setting in the negative phase II NEfERT-T trial ${ }^{40}$, which failed to demonstrate the benefits of neratinib in association with paclitaxel, compared with chemotherapy and trastuzumab. Concerning the subgroup of patients with central nervous system (CNS) disease, neratinib in monotherapy has failed to show substantial efficacy ${ }^{41}$, however, its association with capecitabine in later lines proved a considerable ORR of $32.8 \%$ (95\% CI: 27.1-38.9) ${ }^{39}$.

Pyrotinib is a second-generation, irreversible, pan-HER receptor TKI targeting EGFR, HER-2, and HER4. It was studied in phase II-Chinese study with patients pretreated with trastuzumab, anthracyclines and taxanes. The drug was combined with capecitabine and compared to lapatinib with capecitabine. ${ }^{42}$ ORR was significantly higher in the intervention group ( $78 \%$ vs $57 \%$ [95\% CI: $4.0 \%-38.7 \%$ ]; $\mathrm{p}=0.01$ ). There was an 11 month (18 vs 7) increase in PFS in the pyrotinib group (HR: 0.36 [95\% CI: 0.23-0.58]; $\mathrm{p}<0.001$ ). However, grade 3 or 4 toxicities were higher with pyrotinib combination with mainly diarrhea and hand-foot syndrome, without significant impact in the discontinuation rate compared to the standard arm.

Another phase III, known as PHOEBE trial (patients pretreated with trastuzumab and taxanes) confirmed the longer PFS (12.5 months vs 6.8 months, HR: 0.39 [95\% CI: $0.27-0.56] ; \mathrm{p}<0.0001)$ as well as the manageable toxicity profile of pyrotinib. ${ }^{43}$ To the best of our knowledge, this drug has never been compared to the standard of care in the firstline in the metastatic setting.

Margetuximab. Another potentially useful drug in the therapeutic arsenal against HER-2 positive breast cancer is margetuximab ${ }^{44}$. This anti-HER-2 monoclonal antibody has a similar affinity and specificity to trastuzumab but has a modified $F c$ region. The engineered chimeric $F c$ region has increased binding to CD16A with improved antibody-dependent cell toxicity. Pre-clinical studies showed an enhanced efficacy against, among others, tumors with low expression of HER-2 or those that have developed resistance to trastuzumab treatment.

Clinical efficacy was tested in the phase III SOPHIA clinical trial $^{45}$. Patients with disease progression on at least two antiHER-2 therapies and less than three therapies in the metastatic setting were randomized to chemotherapy in association with margetuximab or trastuzumab. The primary endpoint of PFS was significantly different between the two treatment arms (HR: 0.76 [95\% CI: 0.59-0.98]; $\mathrm{p}=0.03$ ) but 
with a median PFS gain of 0.9 months. Final results for the secondary endpoint (OS) are awaited in 2021 to determine if the relatively marginal PFS gain is translated into OS improvement.

\section{PERSPECTIVES}

The advent of anti-HER-2 has transformed the outlook of HER-2 positive disease and constitutes one of the first examples of precision medicine. While targeting a driving genomic alteration has been achieved in the management of HER-2 positive breast cancer, diverse response to treatment and prognosis can still be observed and could be linked with marked to inter- and intra-tumoral heterogeneity. For instance, subgroup analyses of several randomized clinical trials of anti-HER-2 therapies are consistently showing a trend towards a higher magnitude of OS benefit in patients with hormone receptor (HR) negative/ HER-2 positive disease. ${ }^{22,35,39}$ Moreover, in the PATRICIA phase II trial, assessing the safety of a combination of the CDK4/6 inhibitor palbociclib and trastuzumab (with or without endocrine therapy for hormone sensible $[\mathrm{ER}+]$ cases), Luminal A and B Pam50 intrinsic molecular subtypes ${ }^{46}$ were independently associated with longer PFS than nonluminal tumors ${ }^{47}$. Concerning intra-tumoral heterogeneity, tumor subpopulations with low levels or absence of HER-2 amplification have been identified as potential drivers of resistance to treatment and disease progression in the early and advanced settings. ${ }^{48,49}$

Interestingly, within each molecular subtype, minimal transcriptomic differences (between $0.3 \%$ and $3.9 \%$ of genes differentially expressed), other than HER-2 expression, were observed between HER-2 positive and negative tumors. Furthermore, in HER-2 positive tumors, HER-2 expression is higher in the HER-2 and basal-like molecular subtypes than in Luminal subtypes. ${ }^{50}$

HER-2 expression has been identified as a prognostic biomarker in the CLEOPATRA study ${ }^{51}$, with higher expression levels associated with a better prognosis. The same results were observed in the EMILIA ${ }^{52}$ and TH3RESA trials $s^{53}$. Combining HER-2 expression with molecular subtyping to identify a subgroup of patients (HER-2 molecular subtype and HER-2 high expression) allowed the identification of HER-2 positive early breast cancer that is highly responsive to HER-2 treatment and raises the possibility of chemotherapy de-escalation in this group of patients. Applying these findings to the management of advanced breast cancer may contribute to future de-escalation studies in the advanced setting. ${ }^{54}$

On the other side of the spectrum, the management of HER-2 low tumors (without ERBb2 amplification by in situ hybridization, expressing low protein levels by immunohistochemistry (IHC) is the focus of intense research). This group is marked by biological heterogeneity, with the majority of the tumors being HR-positive (65\%). ${ }^{55}$ Patients with HER-2 low disease have a similar survival profile to those with HER-2 negative disease, independent of HR status. A large phase III trial is ongoing to determine the efficacy of trastuzumab deruxtecan in the advanced HER-2 low setting. ${ }^{32}$

While immunotherapy in general, and immune checkpoint inhibitors in particular, have revolutionized the management of several types of cancer, their use in the management of breast cancer is slowly starting to materialize, particularly for patients with advanced triple-negative breast cancer (TNBC). The focus of extensive research is also how to harness the therapeutical power of immunotherapy to improve the outcomes of patients with HER-2 positive disease.

A retrospective analysis of the CLEOPATRA study showed that high levels of tumor-infiltrating lymphocytes (TILs) were associated with a better prognosis, suggesting that therapies that elicit immunity in HER-2 positive tumors could improve outcomes. ${ }^{56}$ However, the addition of programmed deathligand 1 (PD-L1) inhibitor atezolizumab to trastuzumab emtansine led to more adverse events without improved survival outcomes. ${ }^{57}$ As observed in other immunotherapy trials, a trend towards a benefit was observed in a subgroup of patients with high expression of PD-L1. Similar results have been observed in the phase Ib-II Trial PANACEA, assessing the safety and activity of the pembrolizumab-trastuzumab combination for patients with trastuzumab-resistant HER-2 advanced disease. ${ }^{58}$ Additional trials are ongoing to determine the efficacy of immunotherapy in the immune marker-defined subgroups of patients that could derive the most benefit.

As in other cancer types and subtypes of breast cancer, deescalation trials are increasingly frequent. In various studies in the advanced HER-2 setting, a subset of patients can be long responders. For instance, approximately $16 \%$ of the patients receiving the trastuzumab-pertuzumab maintenance treatment in the CLEOPATRA study are alive and progression-free 8 years after enrolment, despite only $54 \%$ of the included in the study patients being treatment naive. ${ }^{59}$ Similar rates of long responders can also be observed in the EMILIA trial ${ }^{18}$. Current guidelines suggest an anti-HER-2 maintenance treatment until intolerance or disease progression. This strategy may result in increased adverse events and financial toxicity without added benefit for some of the patients that may be long responders. Furthermore, de novo metastatic patients are characterized by longer PFS and lower prevalence of brain metastasis, and they could be the target of initial intense treatment that could prevent the need for indefinite maintenance therapy. ${ }^{60}$

With this in mind, the Translational Breast Cancer Research Consortium will start a phase II clinical trial assessing the safety and efficacy of an approximately 2 -year treatment plan that includes a sequence of several anti-HER-2 treatments and local therapy with patients with treatment naïve advanced breast cancer. Monitoring, by following circulating tumor DNA, will also be performed to determine the potential utility of this biomarker in the follow-up of this particular group of patients. ${ }^{17}$ 
Therapies targeting the CDK4/6 and PIK3CA mutationdependent mTOR signaling activation have transformed the management of metastatic non-HER-2 amplified estrogenpositive disease. Patients with HER-2 amplification were excluded from the SOLAR-1 study ${ }^{61}$ (evaluating the efficacy of PIK3CA inhibitor alpelisib in ER(+)/HER-2(-) metastatic disease), and a dedicated study for patients with HER-2 amplified hormone receptive positive tumors is ongoing (NCT04208178) ${ }^{62}$.

CDK 4/6 inhibitors (e.g., palbociclib, ribociclib and abemaciclib) target cell cycle progression directly in ER+ tumors without retinoblastoma (RB) loss or loss of function. The efficacy of abemaciclib in combination with fulvestrant and trastuzumab as a third or more line of treatment in metastatic ER-positive/HER-2 positive treatment was tested in the monarcHER trial $^{63}$. The triple combination was superior to the chemotherapy and trastuzumab standard of care, resulting in improved PFS at an $80 \%$ significance level (median PFS of 8.3 months vs 5.7 months, HR: 0.67 [95\% CI: $0.45-1.00] ; \mathrm{p}=0.051)$. Surprisingly, the same result was not observed when the study arm of abemaciclib and trastuzumab without fulvestrant was compared with the standard of care, suggesting that endocrine treatment is similar to the HER-2 negative context necessary for optimal CDK4/6 efficacy. ${ }^{64}$
OS results areeagerly awaited to determine the extent to which the identified PFS benefit translates into a longer OS.

\section{CONCLUSIONS}

There is no doubt that significant progress has been achieved in the treatment of HER-2 metastatic breast cancer in the last decades, leading to a clear improvement of prognosis. HER-2 overexpression protein, initially classified as a predictive biomarker of poor prognosis, has now become a targetable molecular alteration, with many HER-2 inhibitors having greatly improved patients' outcomes and long-term survival. Unfortunately, acquired resistance is the main hinder in the course of this disease, leading to tumor progression. More data that will help to understand the resistant mechanisms better are needed and helpful predictive biomarkers to guide the determination of the best and individualized treatment sequence of each patient. Integration of new treatments for specific subgroups will maximize outcomes and patients' quality of life. Many different molecules are actually being evaluated and will undoubtedly change the landscape of treatments of this particular subtype of breast cancer.

\section{CONFLICT OF INTEREST}

The authors declare no conflict of interest.

\section{REFERENCES}

1. Burstein HJ. The Distinctive Nature of HER2-Positive Breast Cancers. $N$ Engl J Med. 2005;353(16):1652-1654. doi:10.1056/NEJMp058197

2. Kennecke H, Yerushalmi R, Woods R, et al. Metastatic Behavior of Breast Cancer Subtypes. J Clin Oncol. 2010;28(20):3271-3277. doi:10.1200/ JCO.2009.25.9820

3. Mendes D, Alves C, Afonso N, et al. The benefit of HER2-targeted therapies on overall survival of patients with metastatic HER2-positive breast cancer - a systematic review. Breast Cancer Res. 2015;17(1):140. doi: 10.1186/s13058-015-0648-2

4. Noone AM, Cronin KA, Altekruse SF, et al. Cancer Incidence and Survival Trends by Subtype Using Data from the Surveillance Epidemiology and End Results Program, 1992-2013. Cancer Epidemiol Biomarkers Prev. 2017;26(4):632-641. doi:10.1158/1055-9965. EPI-16-0520

5. Moasser MM. The oncogene HER2: its signaling and transforming functions and its role in human cancer pathogenesis. Oncogene. 2007;26(45):6469-6487. doi:10.1038/sj.onc. 1210477

6. De P, Hasmann M, Leyland-Jones B. Molecula determinants of trastuzumab efficacy: What is their clinical relevance?. Cancer Treat Rev. 2013;39(8):925934. doi:10.1016/j.ctrv.2013.02.006

7. Slamon DJ, Leyland-Jones B, Shak S, et al. Use of Chemotherapy plus a Monoclonal Antibody against HER2 for Metastatic Breast Cancer That Overexpresses HER2. N Engl J Med. 2001;344(11):783-792. doi:10.1056/NEJM200103153441101

8. Balduzzi S, Mantarro S, Guarneri V, et al. Trastuzumab-containing regimens for metastatic breast cancer. Cochrane Breast Cancer Group, ed. Cochrane Database Syst Rev. 2014;2014(6):CD006242. doi:10.1002/14651858.CD006242.pub2

9. Andersson M, Lidbrink E, Bjerre K, et al. Phase III Randomized Study Comparing Docetaxel Plus Trastuzumab With Vinorelbine Plus Trastuzumab As First-Line Therapy of Metastatic or Locally Advanced Human Epidermal Growth Factor Receptor
2-Positive Breast Cancer: The HERNATA Study. J Clin Oncol. 2011;29(3):264-271. doi:10.1200/ JCO.2010.30.8213

10. Pagani O, Klingbiel D, Ruhstaller T, et al. Do all patients with advanced HER2 positive breast cancer need upfront-chemo when receiving trastuzumab? Randomized phase III trial SAKK 22/99. Ann Oncol. 2017;28(2):305-312. doi:10.1093/annonc/mdw622 11. Swain SM, Baselga J, Kim SB, et al. Pertuzumab, Trastuzumab, and Docetaxel in HER2-Positive Metastatic Breast Cancer. N Engl J Med. 2015;372(8):724734. doi:10.1056/NEJMoa1413513

12. Bachelot T, Ciruelos E, Schneeweiss A, et al. Preliminary safety and efficacy of first-line pertuzumab combined with trastuzumab and taxane therapy for HER2-positive locally recurrent or metastatic breast cancer (PERUSE). Ann Oncol. 2019;30(5):766-773. doi:10.1093/annonc/mdz061

13. Kaufman B, Mackey JR, Clemens MR, et al. Trastuzumab Plus Anastrozole Versus Anastrozole Alone for the Treatment of Postmenopausal Women With Human Epidermal Growth Factor Receptor 2-Positive, Hormone Receptor-Positive Metastatic Breast Cancer: Results From the Randomized Phase III TAnDEM Study. J Clin Oncol. 2009;27(33):55295537. doi:10.1200/JCO.2008.20.6847

14. Rimawi M, Ferrero JM, de la Haba-Rodriguez J, et al. First-Line Trastuzumab Plus an Aromatase Inhibitor, With or Without Pertuzumab, in Human Epidermal Growth Factor Receptor 2-Positive and Hormone Receptor-Positive Metastatic or Locally Advanced Breast Cancer (PERTAIN): A Randomized, OpenLabel Phase II Trial. J Clin Oncol. 2018;36(28):28262835. doi:10.1200/JCO.2017.76.7863

15. LoRusso PM, Weiss D, Guardino E, Girish S, Sliwkowski MX. Trastuzumab Emtansine: A Unique Antibody-Drug Conjugate in Development for $\mathrm{Hu}$ man Epidermal Growth Factor Receptor 2-Positive Cancer. Clin Cancer Res. 2011;17(20):6437-6447. doi:10.1158/1078-0432.CCR-11-0762

16. Perez EA, Barrios C, Eiermann W, et al. Trastuzumab emtansine with or without pertuzumab ver- sus trastuzumab with taxane for human epidermal growth factor receptor 2-positive advanced breast cancer: Final results from MARIANNE. Cancer. 2019;125(22):3974-3984. doi:10.1002/cncr.32392 17. Cardoso F, Paluch-Shimon S, Senkus E, et al. 5th ESO-ESMO international consensus guidelines for advanced breast cancer (ABC 5). Ann Oncol. 2020;31(12):1623-1649. doi:10.1016/j.annonc.2020.09.010

18. Diéras V, Miles D, Verma S, et al. Trastuzumab emtansine versus capecitabine plus lapatinib in patients with previously treated HER2-positive advanced breast cancer (EMILIA): a descriptive analysis of final overall survival results from a randomised, open-label, phase 3 trial. Lancet Oncol. 2017;18(6):732-742. doi:10.1016/S1470-2045(17)30312-1

19. Krop IE, Kim SB, Martin AG, et al. Trastuzumab emtansine versus treatment of physician's choice in patients with previously treated HER2-positive metastatic breast cancer (TH3RESA): final overall survival results from a randomised open-label phase 3 trial. Lancet Oncol. 2017;18(6):743-754. doi:10.1016/ S1470-2045(17)30313-3

20. Konecny GE, Pegram MD, Venkatesan N, et al. Activity of the Dual Kinase Inhibitor Lapatinib (GW572016) against HER-2-Overexpressing and Trastuzumab-Treated Breast Cancer Cells. Cancer Res. 2006;66(3):1630-1639. doi:10.1158/00085472.CAN-05-1182

21. Cameron D, Casey M, Oliva C, Newstat B, Imwalle B, Geyer CE. Lapatinib Plus Capecitabine in Women with HER-2-Positive Advanced Breast Cancer: Final Survival Analysis of a Phase III Randomized Trial. Oncologist. 2010;15(9):924-934. doi:10.1634/ theoncologist.2009-0181

22. Blackwell KL, Burstein HJ, Storniolo AM, et al. Overall Survival Benefit With Lapatinib in Combination With Trastuzumab for Patients With $\mathrm{Hu}$ man Epidermal Growth Factor Receptor 2-Positive Metastatic Breast Cancer: Final Results From the EGF104900 Study. J Clin Oncol. 2012;30(21):25852592. doi:10.1200/JCO.2011.35.6725 
Treatment Landscape of HER-2 Positive Metastatic Breast Cancer

23. Ogitani Y, Aida T, Hagihara K, et al. DS-8201a, A Novel HER2-Targeting ADC with a Novel DNA Topoisomerase I Inhibitor, Demonstrates a Promising Antitumor Efficacy with Differentiation from T-DM1. Clin Cancer Res. 2016;22(20):5097-5108. doi:10.1158/1078-0432.CCR-15-2822 24. Ogitani Y, Hagihara K, Oitate M, Naito H, Agatsuma T. Bystander killing effect of DS-8201a, a novel anti-human epidermal growth factor receptor 2 antibody-drug conjugate, in tumors with human epidermal growth factor receptor 2 heterogeneity. Cancer Sci. 2016;107(7):1039-1046. doi:10.1111/cas. 12966 25. Modi S, Park H, Murthy RK, et al. Antitumor Activity and Safety of Trastuzumab Deruxtecan in Patients With HER2-Low-Expressing Advanced Breast Cancer: Results From a Phase Ib Study. J Clin Oncol. 2020;38(17):1887-1896. doi:10.1200/ JCO.19.02318

26. Modi S, Saura C, Yamashita T, et al. Trastuzumab Deruxtecan in Previously Treated HER2-Positive Breast Cancer. $N$ Engl J Med. 2020;382(7):610-621. doi:10.1056/NEJMoa1914510

27. Cortés J, Kim S, Chung W, et. al. Trastuzumab deruxtecan (T-DXd) vs trastuzumab emtansine (TDM1) in patients (Pts) with HER2 + metastatic breast cancer $(\mathrm{mBC})$ : Results of the randomized phase III DESTINY-Breast03 study. Ann Oncol. 2021; 32 (suppl_5): S1283-S1346.

28. DS-8201a in Pre-treated HER2 Breast Cancer That Cannot be Surgically Removed or Has Spread [DESTINY-Breast02]. ClinicalTrial.gov. [Accessed August 2021]. Available from: https://www.clinicaltrials.gov/ct2/show/NCT03523585.

29. DS-8201a Versus T-DM1 for Human Epidermal Growth Factor Receptor 2 (HER2)-Positive, Unresectable and/or Metastatic Breast Cancer Previously Treated With Trastuzumab and Taxane [DESTINYBreast03]. ClinicalTrial.gov. [Accessed August 2021]. Available from: https://www.clinicaltrials.gov/ct2/ show/NCT03529110.

30. Trastuzumab Deruxtecan (T-DXd) With or Without Pertuzumab Versus Taxane, Trastuzumab and Pertuzumab in HER2-positive Metastatic Breast Cancer (DESTINY-Breast09). ClinicalTrial.gov. [Accessed August 2021]. Available from: https://www. clinicaltrials.gov/ct2/show/NCT04784715.

31. Study of Trastuzumab Deruxtecan (T-DXd) vs Investigator's Choice Chemotherapy in HER2-low, Hormone Receptor Positive, Metastatic Breast Cancer (DB-06). ClinicalTrial.gov. [Accessed August 2021]. Available from: https://www.clinicaltrials. gov/ct2/show/NCT04494425.

32. Trastuzumab Deruxtecan (DS-8201a) Versus Investigator's Choice for HER2-low Breast Cancer That Has Spread or Cannot be Surgically Removed [DESTINY-Breast04]. ClinicalTrial.gov. [Accessed August 2021]. Available from: https://clinicaltrials. gov/ct2/show/NCT03734029.

33. Leyland-Jones B. Human Epidermal Growth Factor Receptor 2-Positive Breast Cancer and Central Nervous System Metastases. J Clin Oncol. 2009;27(31):5278-5286. doi:10.1200/ JCO.2008.19.848

34. Pivot X, Manikhas A, Żurawski B, et al CEREBEL (EGF111438): A Phase III, Randomized, Open-Label Study of Lapatinib Plus Capecitabine Versus Trastuzumab Plus Capecitabine in Patients With Human Epidermal Growth Factor Receptor 2-Positive Metastatic Breast Cancer. J Clin Oncol. 2015;33(14):1564-1573. doi:10.1200/ JCO.2014.57.1794

35. Murthy RK, Loi S, Okines A, et al. Tucatinib, Trastuzumab, and Capecitabine for HER2-Positive Metastatic Breast Cancer. N Engl J Med. 2020;382(7):597609. doi:10.1056/NEJMoa1914609

36. Wu Q, Liao W, Zhang M, Huang J, Zhang P, Li Q. Cost-Effectiveness of Tucatinib in Human Epidermal Growth Factor Receptor 2-Positive Metastatic Breast Cancer From the US and Chinese Perspectives. Front Oncol. 2020;10:1336. doi:10.3389/fonc.2020.01336 37. A Study of Tucatinib Plus Trastuzumab Deruxtecan in HER2+ Breast Cancer (HER2CLIMB-04). ClinicalTrial.gov. [Accessed August 2021]. Avai- lable from: https://clinicaltrials.gov/ct2/show/ NCT04539938.

38. Martin M, Holmes FA, Ejlertsen B, et al. Neratinib after trastuzumab-based adjuvant therapy in HER2-positive breast cancer (ExteNET): 5-year analysis of a randomised, double-blind, placebo-controlled, phase 3 trial. Lancet Oncol. 2017;18(12):16881700. doi:10.1016/S1470-2045(17)30717-9

39. Saura C, Oliveira M, Feng YH, et al. Neratinib Plus Capecitabine Versus Lapatinib Plus Capecitabine in HER2-Positive Metastatic Breast Cancer Previously Treated With $\geq 2$ HER2-Directed Regimens: Phase III NALA Trial. J Clin Oncol. 2020;38(27):3138 3149. doi:10.1200/JCO.20.00147

40. Awada A, Colomer R, Inoue K, et al. Neratinib Plus Paclitaxel vs Trastuzumab Plus Paclitaxel in Previously Untreated Metastatic ERBB2-Positive Breast Cancer: The NEfERT-T Randomized Clinical Trial. JAMA Oncol. 2016;2(12):1557. doi:10.1001/jamaoncol.2016.0237

41. Freedman RA, Gelman RS, Wefel JS, et al. Translational Breast Cancer Research Consortium (TBCRC) 022: A Phase II Trial of Neratinib for Patients With Human Epidermal Growth Factor Receptor 2-Positive Breast Cancer and Brain Metastases. J Clin Oncol. 2016;34(9):945-952. doi:10.1200/ CO.2015.63.0343

42. Ma F, Ouyang Q, Li W, et al. Pyrotinib or Lapatinib Combined With Capecitabine in HER2-Positive Metastatic Breast Cancer With Prior Taxanes, Anthracyclines, and/or Trastuzumab: A Randomized, Phase II Study. J Clin Oncol. 2019;37(29):2610-2619. doi:10.1200/JCO.19.00108

43. Xu B, Yan M, Ma F, et al. Pyrotinib plus capecitabine versus lapatinib plus capecitabine for the treatmen of HER2-positive metastatic breast cancer ( $\mathrm{PHOE}$ $\mathrm{BE})$ : a multicentre, open-label, randomised, controlled, phase 3 trial. Lancet Oncol. 2021;22(3):351-360. doi:10.1016/S1470-2045(20)30702-6

44. Nordstrom JL, Gorlatov S, Zhang W, et al. Anti-tumor activity and toxicokinetics analysis of MGAH22, an anti-HER2 monoclonal antibody with enhanced Fcg receptor binding properties. Breast Cancer Res. 2011;13(6):R123. doi:10.1186/bcr3069 5. Rugo HS, Im S-A, Cardoso F, et al. Efficacy of Margetuximab vs Trastuzumab in Patients With Pretreated ERBB2-Positive Advanced Breast Cancer A Phase 3 Randomized Clinical Trial. JAMA Oncol. 2021;7(4):573. doi:10.1001/jamaoncol.2020.7932

46. Sorlie T, Perou CM, Tibshirani R, et al. Gene expression patterns of breast carcinomas distinguish tumor subclasses with clinical implications. Proc Natl Acad Sci U S A. 2001;98(19):10869-10874. doi:10.1073/pnas. 191367098

47. Ciruelos E, Villagrasa P, Pascual T, et al. Palbociclib and Trastuzumab in HER2-Positive Advanced Breast Cancer: Results from the Phase II SOLTI-1303 PATRICIA Trial. Clin Cancer Res. 2020;26(22):5820-5829. doi:10.1158/1078-0432. CCR-20-0844

48. Lee HJ, Seo AN, Kim EJ, et al. HER2 Heterogeneity Affects Trastuzumab Responses and Surviva in Patients With HER2-Positive Metastatic Breast Cancer. Am J Clin Pathol. 2014;142(6):755-766. doi:10.1309/AJCPIRL4GUVGK3YX

49. Metzger Filho O, Viale G, Stein S, et al. Impact of HER2 heterogeneity on treatment response of early-stage HER2-positive breast cancer: phase II neoadjuvant clinical trial of T-DM1 combined with pertuzumab. Cancer Discov. Published online May 3, 2021:candisc.1557.2020. doi:10.1158/2159-8290. CD-20-1557

0. Prat A, Carey LA, Adamo B, et al. Molecular Features and Survival Outcomes of the Intrinsic Subtypes Within HER2-Positive Breast Cancer. J Natl Cancer Inst. 2014;106(8):dju152. doi:10.1093/jnci/dju152 1. Baselga J, Cortés J, Im SA, et al. Biomarker Analyses in CLEOPATRA: A Phase III, Placebo-Controlled Study of Pertuzumab in Human Epidermal Growth Factor Receptor 2-Positive, First-Line Metastatic Breast Cancer. J Clin Oncol. 2014;32(33):3753-3761. doi:10.1200/JCO.2013.54.5384

52. Baselga J, Lewis Phillips GD, Verma S, et al. Rela- tionship between Tumor Biomarkers and Efficacy in EMILIA, a Phase III Study of Trastuzumab Emtansin in HER2-Positive Metastatic Breast Cancer. Clin Cancer Res. 2016;22(15):3755-3763. doi:10.1158/10780432.CCR-15-2499

53. Kim SB, Wildiers H, Krop IE, et al. Relationship between tumor biomarkers and efficacy in TH3RESA a phase III study of trastuzumab emtansine (T-DM1) vs. treatment of physician's choice in previously treated HER2-positive advanced breast cancer. Int J Cancer 2016;139(10):2336-2342. doi:10.1002/ijc.30276 54. Prat A, Pascual T, De Angelis C, et al. HER2Enriched Subtype and ERBB2 Expression in HER2 Positive Breast Cancer Treated with Dual HER2 Blockade. J Natl Cancer Inst. 2020;112(1):46-54. doi:10.1093/jnci/djz042

55. Schettini F, Chic N, Brasó-Maristany F, et al. Clinical, pathological, and PAM50 gene expression features of HER2-low breast cancer. NPJ Breast Cancer. 2021;7(1):1. doi:10.1038/s41523-020-00208-2 56. Luen SJ, Salgado R, Fox S, et al. Tumour-infiltrating lymphocytes in advanced HER2-positive breast cancer treated with pertuzumab or placebo in addition to trastuzumab and docetaxel: a retrospective analysis of the CLEOPATRA study. Lan cet Oncol. 2017;18(1):52-62. doi:10.1016/S14702045(16)30631-3

57. Emens LA, Esteva FJ, Beresford M, et al. Trastuzumab emtansine plus atezolizumab versus trastuzumab emtansine plus placebo in previously treated, HER2-positive advanced breast cancer (KATE2): phase 2, multicentre, randomised, double-blind trial. Lancet Oncol. 2020;21(10):1283-1295. doi:10.1016 S1470-2045(20)30465-4

58. Loi S, Giobbie-Hurder A, Gombos A, et al Pembrolizumab plus trastuzumab in trastuzumab-re sistant, advanced, HER2-positive breast cancer (PA NACEA): a single-arm, multicentre, phase $1 \mathrm{~b}-2$ trial. Lancet Oncol. 2019;20(3):371-382. doi:10.1016/ S1470-2045(18)30812-X

59. Swain SM, Miles D, Kim SB, et al. Pertuzumab, trastuzumab, and docetaxel for HER2 positive metastatic breast cancer (CLEOPAT RA): end-of-study results from a double-blind, randomised, placebo-controlled, phase 3 study. Lancet Oncol. 2020;21(4):519-530, doi:10.1016/S1470 2045(19)30863-0

60. Tripathy D, Brufsky A, Cobleigh M, et al. De Novo Versus Recurrent HER2-Positive Metastatic Breast Cancer: Patient Characteristics, Treatment, and Survival from the SystHERs Registry. Oncolo gist. 2020;25(2):e214-e222. doi:10.1634/theoncologist.2019-0446

61. André F, Ciruelos EM, Juric D, et al. Alpelisib plus fulvestrant for PIK3CA-mutated, hormone receptorpositive, human epidermal growth factor receptor-2 negative advanced breast cancer: final overall survival results from SOLAR-1. Ann Oncol. 2021;32(2):208 217. doi:10.1016/j.annonc.2020.11.011

62. Efficacy of Different Treatment Regimens With Chitosan-N-Acetylcysteine in Moderate-to-severe Dry Eye Disease. ClinicalTrial.gov. [Accessed August 2021]. Available from: https://www.clinicaltrials. gov/ct2/show/NCT04208178.

63. Tolaney SM, Wardley AM, Zambelli S, et al. Abemaciclib plus trastuzumab with or without fulvestrant versus trastuzumab plus standard-of-care chemotherapy in women with hormone receptor-positive, HER2-positive advanced breast cancer (monarcHER): a randomised, open-label, phase 2 trial. Lancet Oncol. 2020;21(6):763-775. doi:10.1016/ S1470-2045(20)30112-1

64. Malorni L, Curigliano G, Minisini AM, et al. Palbociclib as single agent or in combination with the endocrine therapy received before disease progression for estrogen receptor-positive, HER2-negative metastatic breast cancer: TREnd trial. Ann Oncol. 2018;29(8):1748-1754. doi:10.1093/annonc/ mdy 214 